\title{
A diffusion model for the development of a boundary layer in lakes
}

\author{
Frank Peeters, Gabriel Piepke and Manuel Gloor \\ Swiss Federal Institute of Technology (ETH) and Swiss Federal Institute for Environmental \\ Science and Technology (EAWAG), Department of Environmental Physics and Department \\ of Computer and System Sciences, CH-8600 Dübendorf, Switzerland
}

Key words: Mixing model, diffusion, boundary layer, boundary mixing, diapycnal mixing.

\begin{abstract}
The development of a boundary layer characterised by very low gradients in temperature and salinity near the bottom boundary of a lake does not necessarily imply an increase in diapycnal mixing within the boundary layer. The results of a quasi three-dimensional diffusion model for a basin with sloping boundaries demonstrate that in lakes a boundary layer also develops when the diapycnal diffusivity is chosen to be constant.

In the model mixing is assumed to be anisotropic and is described as an isopycnal and diapycnal turbulent diffusion process. Advective transport is not considered. Therefore, the model is restricted to the description of the purely diffusive response of a lake. It should be regarded as a contribution to the discussion of boundary mixing and not as a complete mixing model for a specific lake. The isopycnal and diapycnal turbulent diffusion coefficients are presumed to be constant in space and time. The direction of isopycnal and diapycnal density flux changes with time since mixing of the density distribution influences the orientations of the isopycnals. This interaction between mixing process and density distribution is accounted for by the model.

According to the model the density distribution, and therefore the development of a boundary layer, only depends on diapycnal mixing while the distribution of a passive tracer depends on both, isopycnal and diapycnal mixing. The application of the model to the subalpine Lake Alpnach demonstrates that a simple diffusion model is sufficient to predict the development of a boundary layer. Considering that the model does not include advective processes and that diffusivities have been assumed to be constant in space and time, the structure of the boundary layer predicted agrees surprisingly well with experimental data.
\end{abstract}

\section{Introduction}

In natural waters turbulent mixing is one of the major processes transporting dissolved substances and suspended particles. The lake sediments are source and sink for biological and chemical components. Therefore, mixing near the lake boundaries is of particular importance to the distribution of bio-geochemical substances, e.g. phosphate and oxygen, and thus to the ecology of the entire lake. 
Often, a drastic decrease in the vertical gradients of density and temperature can be observed near the bottom boundary in lakes (Gloor et al., 1994). In this paper we call this region of low gradients the boundary layer.

In the literature vertical diffusion within the boundary layer has been shown to be large compared to the open water (Ivey, 1987 and references therein for experiments in the ocean, and Wüest et al., 1996b in lakes). The influence of this large vertical boundary mixing on the total effective vertical flux in the ocean has been investigated by several researchers (Armi, 1978; Garrett, 1990; Woods, 1991). Note however, that large vertical mixing within the boundary does not necessarily imply large diapycnal mixing since the diapycnal direction is not parallel to the vertical direction within the boundary layer.

The aim of this work is to show that in lakes a simple diffusion model with spatially and temporally constant diapycnal and isopycnal diffusion coefficients can be sufficient to predict a boundary layer, the structure of which is in good qualitative agreement with experimental data. Large vertical mixing within the boundary layer compared to the open water will be shown to be consistent with the model, although isopycnal and diapycnal diffusion coefficients are spatially constant. In the natural environment additional diapycnal mixing might be produced near the lake boundary either by the increase in the velocity shear due to the boundary condition for velocity (e.g. Armi, 1978), by bottom reflection of internal waves (Ericksen, 1985), or by secondary circulation (see Garrett, 1990 and references therein). However, we will show that an increase of diapycnal mixing is not a requirement for the description of the small vertical gradients in density, temperature, and salinity commonly observed close to the sediment.

For isotropic mixing and impermeable walls Wunsch (1970) and Phillips (1970) have shown that the density distribution resulting from the mixing process gives rise to advection. This so-called secondary circulation has been studied theoretically for the boundary layer in the ocean by numerous researchers (see Garrett et al., 1993 and references therein). However, the theoretical models which mostly focus on the boundary layer alone and assume isotropic mixing have only rarely been applied for the comparison with experimental measurements of the boundary layer. To our knowledge, a circulation model that includes secondary circulation and describes the development in time of the entire density structure (and as part of this the development of a boundary layer) of a closed basin under natural conditions does not exist.

The model presented here is purely diffusive and does not consider advective processes including secondary circulation. Thus, it should be regarded as a first order approach in the analysis of the boundary layer development in lakes. The model however allows for anisotropic diffusive mixing and does not focus on the boundary layer alone, but includes the entire water body of a closed basin with sloping boundaries, i.e. Lake Alpnach. As will be shown below our simplified purely diffusive model predicts the development of a boundary layer the rough structure of which agrees surprisingly well with the experimental data (e.g. thickness of the boundary layer). This result is remarkable considering the absence of advection in our model.

In the following we present the experimental technique employed and the measurements obtained. Subsequently, the simplified diffusion model for a lake with sloping boundaries is developed. This is followed by a presentation and discussion of the results of the model. 


\section{Measurements}

Experiments were conducted in Lake Alpnach, a subalpine lake in Switzerland with a surface area of $4.76 \mathrm{~km}^{2}$ and a maximum depth of approximately $33.5 \mathrm{~m}$. At different times in 1992 vertical profiles of temperature, conductivity and turbidity were measured along transects perpendicular to the isopleth. The CTDprobe employed (Multisonde MS 040, Meerestechnik Elektronik GmbH) provided a resolution of $0.01 \mathrm{dbar}$ (pressure), $0.001{ }^{\circ} \mathrm{C}$ (temperature), $0.1 \mu \mathrm{Scm}^{-1}$ (conductivity) and $0.1 \%$ (turbidity). Salinity was calculated from the conductivity measurements using the method outlined by Wüest et al. (1996a). The resolution of salinity is approximately $1 \cdot 10^{-4} \mathrm{~g} / \mathrm{kg}$. The logarithm of turbidity is approximately proportional to the concentration of suspended particles. Since turbidity depends on the distribution of particle size and is not sensitive to the density of the particles an absolute calibration of sediment load as function of turbidity is difficult. To estimate the concentration of suspended particles we have calibrated the turbidity sensor by resuspending the top layer of a sediment core taken in 1992 in Lake Alpnach. According to this calibration a turbidity of $32 \%$, which is typical for the boundary layer corresponds to $3.1 \mathrm{mg} \mathrm{l}^{-1}$ of suspended particles. Because of the mounting of the sensors measurements of turbidity, and thus of suspended particles, are not available for the region $0-20 \mathrm{~cm}$ above the sediment. The ship position was determined with an accuracy of $\pm 1 \mathrm{~m}$ using a microwave positioning system (Trisponder Systems, Del Norte Technology Inc.).

In the vertical direction temperature, salinity and turbidity are almost constant near the lake boundary (Fig. 1, shaded area). Above this "boundary layer" of about $1 \mathrm{~m}$ thickness the gradients in temperature and salinity increase and turbidity shows a sharp drop to smaller values. These data stem from an experiment conducted in November, 1992. However, a similar structure of the vertical distribution of the parameters measured can be observed throughout the year. Figure 2 shows vertical temperature profiles from May and October in 1992. The thickness of the boundary layer increases with the depth of the bottom boundary, i.e. towards the centre of the lake (Fig. 2). The profiles from May were taken in a period of less than 20 minutes.

Density $\rho$, thermal and haline expansion coefficients ( $\alpha$ and $\beta$, respectively), and stability $\left(N^{2}\right)$ were calculated from temperature $T$ and salinity $S$ by the formulas given in Chen and Millero (1986). Throughout this paper density is taken at the pressure at lake surface. The definitions of $\alpha, \beta$ and $N^{2}$ are (the adiabatic temperature gradient is very small and has therefore been neglected):

$$
\begin{aligned}
& \alpha \equiv-\begin{array}{ll}
1 & \partial \rho \\
\rho & \partial T
\end{array} \\
& \beta \equiv \begin{array}{ll}
1 & \partial \rho \\
\rho & \partial S
\end{array} \\
& N^{2} \equiv g \cdot\left(\alpha \cdot \frac{d T}{d z}-\beta \cdot \frac{d S}{d z}\right)=N_{T}^{2}+N_{S}^{2}
\end{aligned}
$$




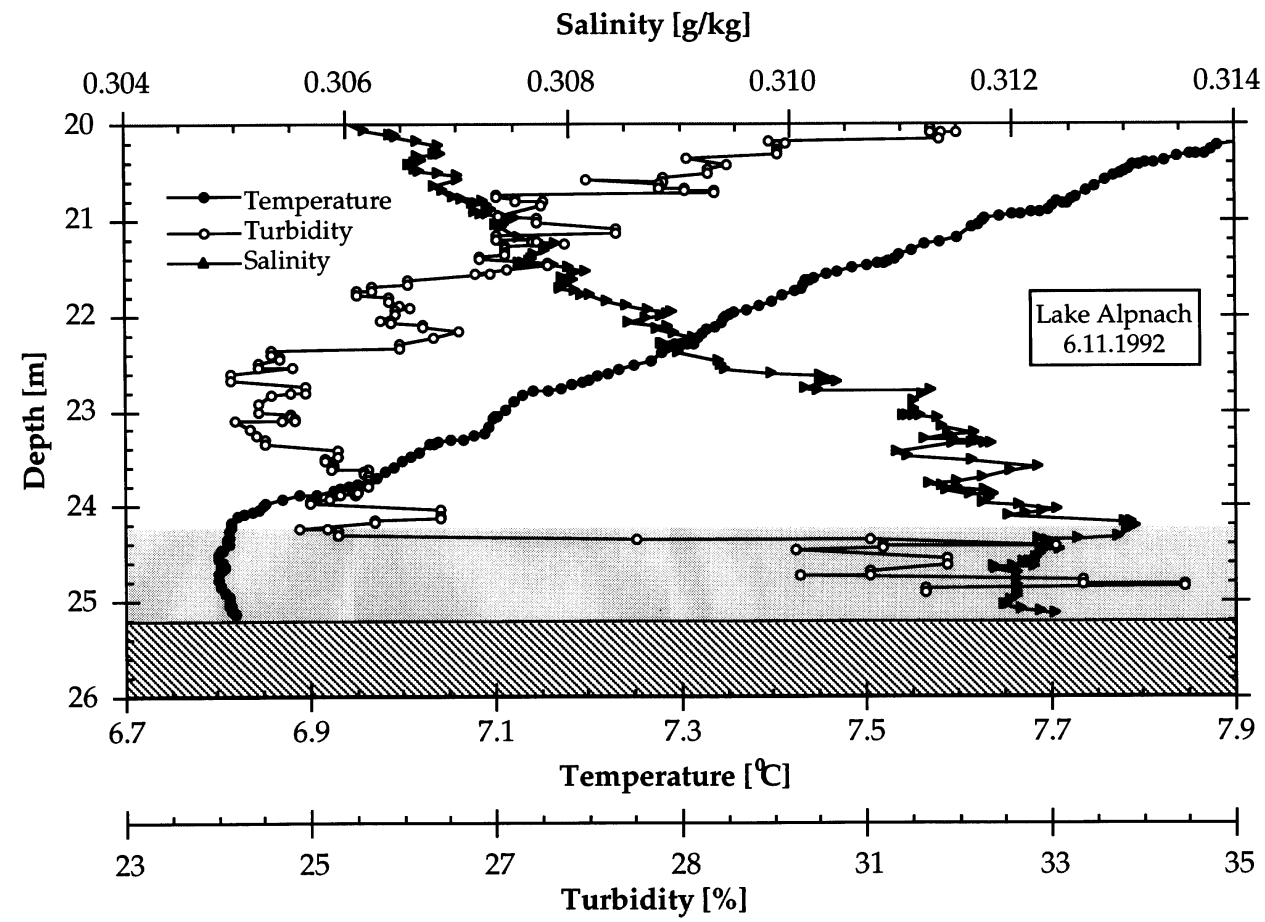

Figure 1. Vertical profiles of temperature, salinity and turbidity in Lake Alpnach taken in November. The grey area indicates the region which we refer to as boundary layer

and $z$ is defined to be positive in the upward direction. The stability $N^{2}$ comprises of two components: the stabilising contributions due to firstly the gradient in temperature $N_{T}^{2}=g \cdot \alpha \cdot d T / d z$ and secondly due to the gradient in salinity $N_{S}^{2}=-g \cdot \beta \cdot d S / d z$. Stability is, in good approximation, proportional to the density gradient.

Similar to temperature and salinity the density of the water column is approximately constant in a region near the lake boundary (shaded area in Fig. 3a). A distinct boundary layer can be identified. The density profile is almost entirely determined by the vertical distribution of temperature. This is confirmed by the profile of stability (Fig. 3b) which is nearly independent of the stabilising contribution of salinity $\left(N_{S}^{2}\right)$. The vertical distribution of temperature dominates the vertical structure of stability ( $N_{T}^{2}$ in Fig. 3b). The instability just above the lake boundary is of the order $-1 \cdot 10^{-5} \mathrm{~s}^{-2}$. Assuming that our calibration of turbidity is correct, an increase of turbidity from 31 to $34 \%$ within $0.5 \mathrm{~m}$ would indicate a gradient of the concentration of suspended particles of about $1 \mathrm{mg} \mathrm{l}^{-1} \mathrm{~m}^{-1}$ which is sufficient to compensate the instability near the lake boundary. However, this argument is not entirely conclusive since the gradient of turbidity is not smooth but changes rapidly within the boundary layer. Note that the negative stratification of temperature in the bottom boundary layer causing the instability discussed above did only occur in 

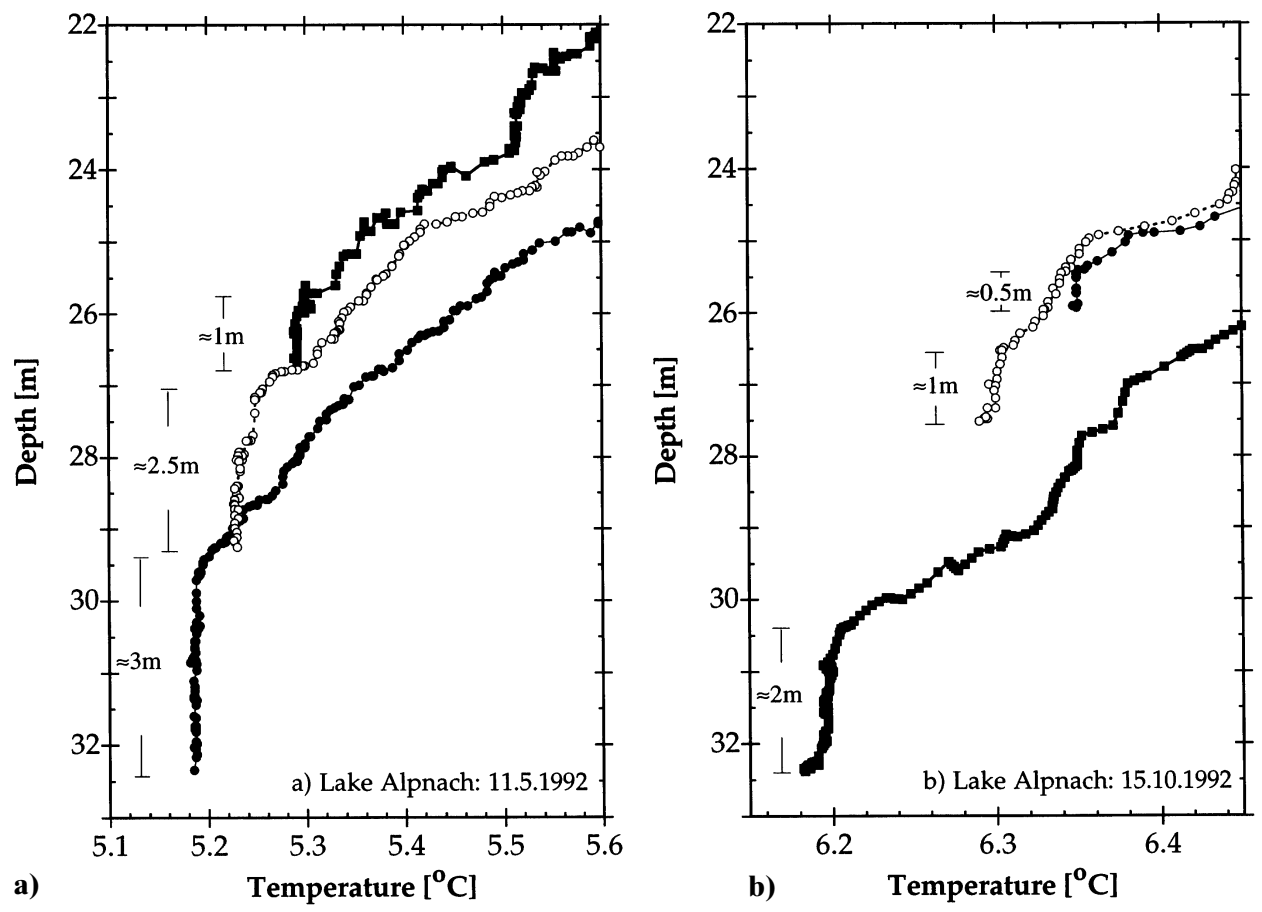

Figure 2. Vertical profiles of temperature from sites of various depths taken in Lake Alpnach in May 1992 (a) and October 1992 (b). The bars indicate the approximate boundary layer thickness

November. In May, July and October 1992 the entire water column was stable (see e.g. the temperature profiles in Fig. 2).

A detailed two-dimensional picture of the boundary layer is shown in Fig. 4. The spatial distance between CTD-casts (small dashed lines) is less than $10 \mathrm{~m}$. The time period required to measure all profiles was less than 15 minutes. Thus, variations of the boundary layer thickness due to internal seiche motion can be neglected (during the summer months the period of the first vertical mode is typically larger than 7 hours; Münnich et al., 1992). The structures of the density and temperature distribution (Fig. 4a and b) agree with each other, indicating that in Lake Alpnach salinity does not play an important role in the development of the boundary layer. The boundary layer at about $25 \mathrm{~m}$ depth is approximately $1 \mathrm{~m}$ thick and increases slightly at larger depth. Within the boundary layer significant horizontal gradients of density and temperature exist.

Within the boundary layer the diapycnal direction is almost horizontal and not vertical as in the open water. Therefore, the small vertical density gradients close to the sediment cannot serve as an indication of large diapycnal mixing within the boundary layer.

Since temperature is not a passive tracer the diffusion of heat causes the direction of isopycnal and diapycnal mixing to change with time. The modifications 


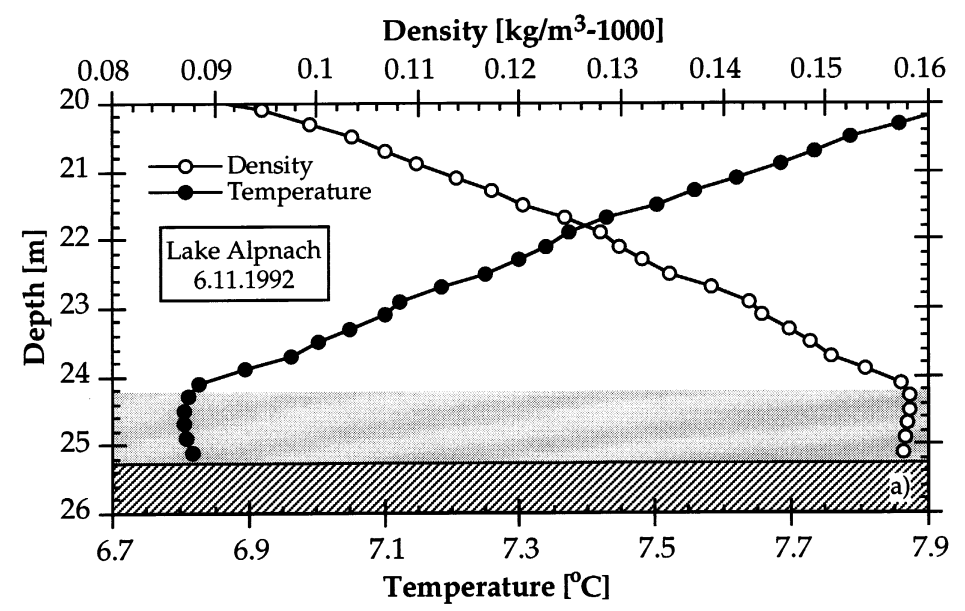

b)

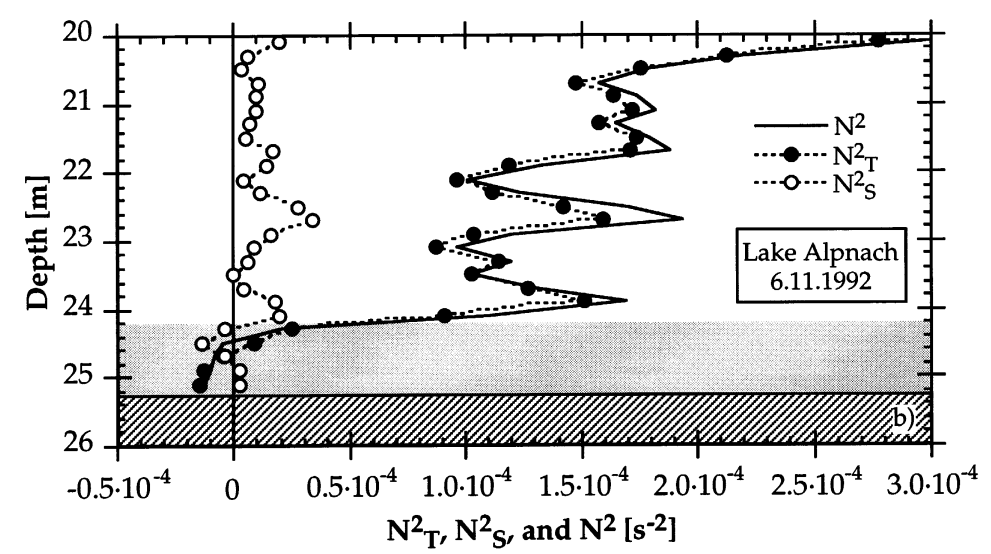

Figure 3. The water density profile (a) and stability (b) in Lake Alpnach observed in November 1992. The stabilising contribution due to the salinity gradient $N_{S}^{2}$ is small compared to the influence of the temperature gradient $N_{T}^{2}$

imposed on the diffusion equation by the development of the density distribution is accounted for in the simple diffusion model discussed below.

\section{Model}

In the following we assume that the transport of dissolved substances in a lake can be described as an isopycnal and diapycnal turbulent diffusion process. Advective processes are neglected. Diapycnal turbulent diffusion is modelled as usual in analogy to molecular diffusion by the Fickian law. In the absence of large scale advection isopycnal transport can be described in the same way (Carter and Okubo, 1965; Peeters et al., 1996). 


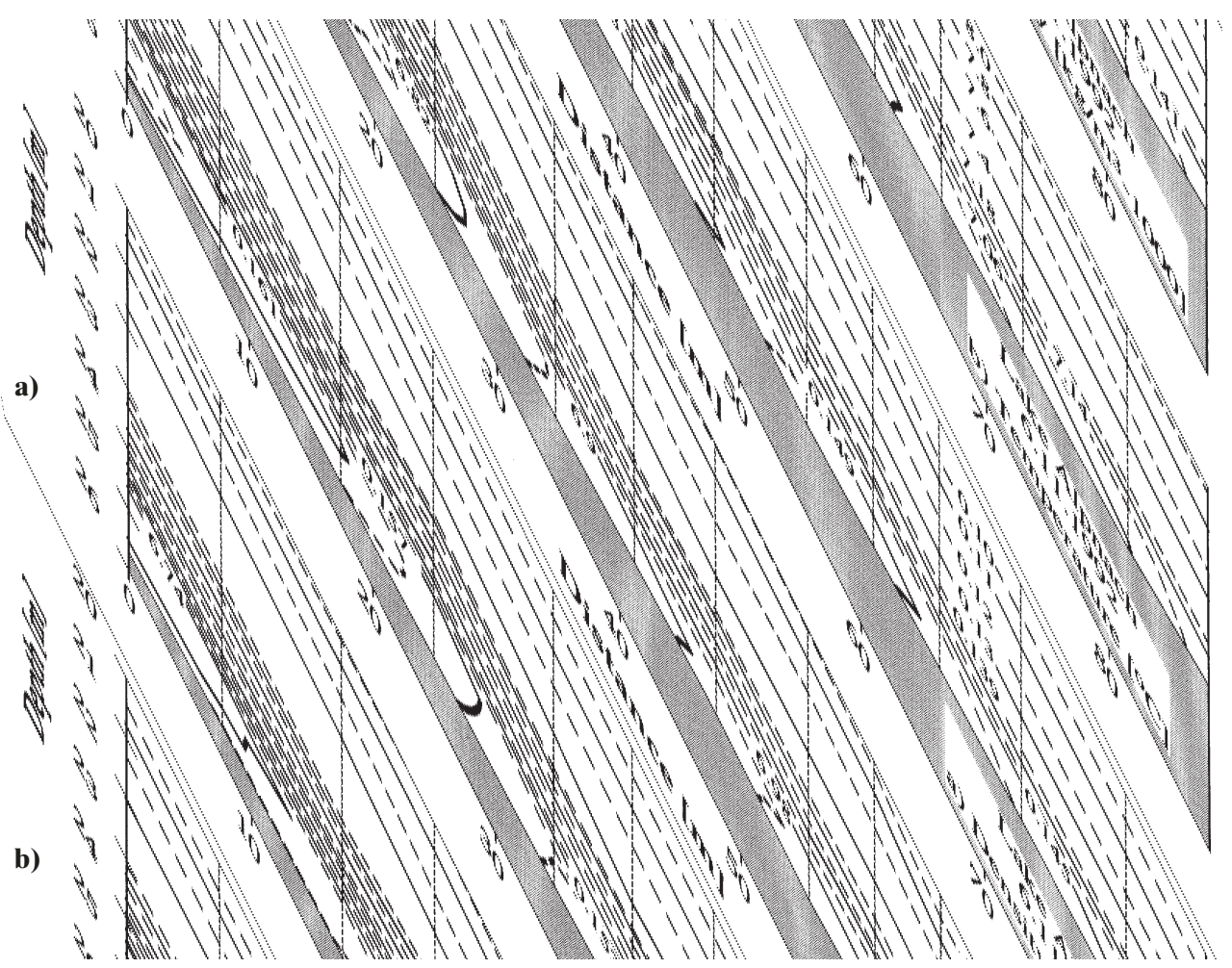

Figure 4. Observed two dimensional distribution of density (a) and temperature (b) near the lake boundary. Thin vertical dashed lines indicate the position of the CTD-casts

\subsection{Turbulent flux}

At first we will consider a two-dimensional basin. The coordinates of the iso-diapycnal coordinate system will be designated by $(\tilde{x}, \tilde{z})$ and the Cartesian coordinates by $(x, z)$. The $z$-coordinate is chosen to be positive in the upward direction. In the isodiapycnal coordinate system the diffusion tensor $\boldsymbol{K}_{\text {iso }}$ is diagonal:

$$
\boldsymbol{K}_{\text {iso }}=\left(\begin{array}{cc}
K_{i} & 0 \\
0 & K_{d}
\end{array}\right),
$$

where $K_{i}$ and $K_{d}$ represent the isopycnal and diapycnal diffusion coefficients, respectively. The turbulent flux $\vec{F}$ of a substance with concentration $C(x, z)$ is given by:

$$
\vec{F}=-\boldsymbol{K}_{\text {iso }} \cdot \vec{\nabla}_{\text {iso }} C=-\left(\begin{array}{cc}
K_{i} & 0 \\
0 & K_{d}
\end{array}\right) \cdot\left(\begin{array}{c}
\partial C \\
\partial \tilde{x} \\
\partial C \\
\partial \tilde{z}
\end{array}\right)
$$


If the isopycnals are horizontal the diffusion tensor $\boldsymbol{K}_{x, z}$ in the Cartesian coordinate system is diagonal too:

$$
\vec{F}=-\left(K_{i} \frac{\partial C}{\partial x} \vec{e}_{x}+K_{d} \frac{\partial C}{d z} \vec{e}_{z}\right)=\boldsymbol{K}_{x, z} \cdot \vec{\nabla}_{x, z} C .
$$

Note that in the anisotropic case $\left(K_{i} \neq K_{d}\right)$ turbulent transport is not necessarily parallel to the gradient of the concentration.

In a basin with sloping boundaries the isopycnals are usually bent downwards and almost vertical near the bottom boundary. The reason for this behaviour lies in the boundary condition for the turbulent flux. The turbulent flux into the basin walls has to vanish if the walls are impermeable as commonly assumed. If the isopycnals are bent the diffusion tensor in the $(x, z)$ coordinate system cannot be diagonal at all locations.

Along the isopycnals $\frac{d \rho}{d \tilde{x}}=0$ applies, where $\tilde{x}$ is parallel to the direction of the isopycnal. The orientation of an isopycnal relative to the Cartesian coordinate system can be described by the angle $\beta(x, z)$ :

$$
\begin{aligned}
& \left(\begin{array}{l}
x \\
z
\end{array}\right)=\left(\begin{array}{c}
\tilde{x} \cos \beta+\tilde{z} \sin \beta \\
-\tilde{x} \sin \beta+\tilde{z} \cos \beta
\end{array}\right), \\
& 0=\begin{array}{l}
d \rho \\
d \tilde{x}
\end{array}=\begin{array}{l}
\partial \rho d x d \tilde{x} \\
\partial x d \rho d z \\
\partial z d \tilde{x}
\end{array}=\begin{array}{l}
\partial \rho \\
\partial x
\end{array} \cos \beta-\frac{\partial \rho}{\partial z} \sin \beta \\
& \left.\Rightarrow \beta=\arctan \left\{\begin{array}{l}
\partial \rho\left(\begin{array}{l}
\partial \rho \\
\partial x
\end{array}\right)-1 \\
\partial z
\end{array}\right)^{-1}\right\}
\end{aligned}
$$

Following Redi (1982) the diffuson tensor in Cartesian coordinates can be calculated by rotating the diffusion tensor given in iso-diapycnal coordinates:

$$
\begin{aligned}
& \boldsymbol{K}_{x, z}=\boldsymbol{R} \boldsymbol{K}_{\text {iso }} \boldsymbol{R}^{-1} \\
& \Rightarrow \boldsymbol{K}_{x, z}=\left(\begin{array}{ll}
K_{i} \cos ^{2} \beta+K_{d} \sin ^{2} \beta & \cos \beta \sin \beta\left(K_{d}-K_{i}\right) \\
\cos \beta \sin \beta\left(K_{d}-K_{i}\right) & K_{i} \sin ^{2} \beta+K_{d} \cos ^{2} \beta
\end{array}\right)
\end{aligned}
$$

From equation 6 the turbulent flux in Cartesian coordinates of a substance with concentration $C(x, z)$ can be derived:

$$
\begin{aligned}
& \vec{F}_{x, z}=-\boldsymbol{K}_{x, z} \vec{\nabla}_{x, z} C \Rightarrow \\
& \vec{F}_{x, z}=-\left(\begin{array}{l}
K_{i}\left(\begin{array}{l}
\cos ^{2} \beta \frac{\partial C}{\partial x}-\sin \beta \cos \beta \\
\partial C
\end{array}\right)+K_{d}\left(\sin ^{2} \beta \frac{\partial C}{\partial x}+\sin \beta \cos \beta \frac{\partial C}{\partial z}\right) \\
K_{i}\left(-\sin \beta \cos \beta \frac{\partial C}{\partial x}+\sin ^{2} \beta \frac{\partial C}{\partial z}\right)+K_{d}\left(\sin \beta \cos \beta \frac{\partial C}{\partial x}+\cos ^{2} \beta \frac{\partial C}{\partial z}\right)
\end{array}\right) .
\end{aligned}
$$




\subsection{Transport of heat if temperature determines the density distribution}

Consider the transport of heat in a lake in which the distribution of density is entirely determined by the temperature distribution. This assumes that the influence of salinity is negligible, i.e. that salinity is approximately constant in space and time. Lake Alpnach might serve as an example for such a situation since salinity has no significant effect on the density distribution: From Fig. 3 b one can see that the density ratio $N_{S}^{2} / N_{T}^{2}$ is about $1: 10$ throughout the stratified water body, indicating that the influence of salinity on the density gradient is about ten times smaller than that of temperature. Therefore, in our "first order" approach salinity can safely be neglected. Under the conditions assumed above $\rho(x, y, z)=\rho(T(x, y, z))$ and the isopycnals and the isotherms have to be parallel ( $\tilde{x}$ is along the isopycnal):

$$
0=\frac{d \rho}{d \tilde{x}}=\begin{aligned}
& \partial \rho d T \\
& \partial T d \tilde{x}
\end{aligned} \Rightarrow \begin{aligned}
& d T \\
& d \tilde{x}
\end{aligned}=0 .
$$

\section{(a) Transport in two dimensions}

In the following we neglect the effect of pressure on temperature because this effect is very small in most lakes due to their shallow depth (e.g. in Lake Alpnach the difference between potential and in situ temperature is less than $0.001{ }^{\circ} \mathrm{C}$ ). Under the condition above the conservation of heat can be written as a conservation equation for temperature (e.g. Pedlosky, 1987). The turbulent flux of temperature $\vec{F}_{x, z}^{T}$ is given by:

$$
\vec{F}_{x, z}^{T}=-\boldsymbol{K}_{x, z} \cdot \vec{\nabla} T
$$

where $\boldsymbol{K}_{x, z}$ is given by equation 6 . Thus, the turbulent heat flux $\vec{F}_{x, z}^{T}$ can be described by equation 7 with $C$ representing $T$. Since the isotherms and the isopycnals are parallel (equation 8 ) $\vec{F}_{x, z}^{T}$ can be simplified by combining equations 5 and 7 . As result, the turbulent flux of temperature can be written as:

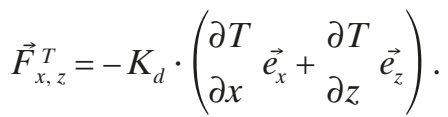

Using equation 10 the balance equation for temperature is given by

$$
\begin{aligned}
& \partial T \\
& \partial t
\end{aligned}=-\vec{\nabla}_{x, z} \vec{F}_{x, z}^{T}=K_{d}\left(\begin{array}{l}
\partial^{2} T \\
\partial x^{2}
\end{array}+\begin{array}{l}
\partial^{2} T \\
\partial z^{2}
\end{array}\right)
$$

This diffusion equation is equivalent to one for isotropic mixing. The temperature balance is independent of isopycnal diffusion. Therefore, the distribution of temperature, and also that of density, is only determined by diapycnal diffusion and the initial and boundary conditions. Note that in general, especially for the flux of passive tracers, equation 7 applies and consequently isopycnal mixing plays an important role for the spatial distribution of the tracer concentration. 


\section{b) Three-dimensional model for a radially symmetric lake}

In a basin which is radially symmetric relative to the vertical it is convenient to use cylindrical coordinates $(r, \varphi, z)$ for the description of concentration distributions and the turbulent flux. The $z$-axis is chosen to correspond to the axis of symmetry. If in addition to the lake geometry initial and boundary conditions are radially symmetric the temperature distribution $T(r, \varphi, z)$ has to be independent of $\varphi$ :

$$
\begin{aligned}
& d T \\
& d \varphi
\end{aligned}=0
$$

In this case the turbulent flux of temperature can be described by equation 10 where the $x$-coordinate has to be replaced by the $r$-coordinate. The balance equation is given by:

$$
\begin{aligned}
& \partial T \\
& \partial t
\end{aligned}=K_{d}\left(\begin{array}{ll}
1 & \partial \\
r & \partial r
\end{array}\left(\begin{array}{r}
r T \\
\partial r
\end{array}\right)+\begin{array}{l}
\partial^{2} T \\
\partial z^{2}
\end{array}\right) .
$$

The temperature balance is independent of the isopycnal diffusion as already discussed above.

\section{c) Initial and boundary conditions}

The turbulent flux perpendicular to the bottom boundary of a basin has to vanish. In general this results in the boundary condition:

$$
0=\vec{F}_{r, z} \cdot \vec{e}_{s}=\left(\boldsymbol{K}_{r, z} \vec{\nabla}_{r, z} C\right) \cdot \vec{e}_{s}
$$

where $\vec{e}_{s}=\left(\begin{array}{c}-\sin \gamma \\ \cos \gamma\end{array}\right)$ is the unit vector perpendicular to the bottom boundary and $\gamma(r, z)$ the angle between bottom boundary and the $r$-axis.

In the special case of temperature transport and constant isopycnal and diapycnal diffusion coefficients the boundary condition is given by:

$$
\begin{aligned}
& K_{d}\left(-\frac{\partial T}{\partial r} \sin \gamma+\frac{\partial T}{\partial z} \cos \gamma\right)=0 \\
& \Rightarrow \underset{\partial r}{\partial T} \sin \gamma={ }_{\partial z}^{\partial T} \cos \gamma .
\end{aligned}
$$

This implies that even if turbulent mixing is anisotropic $\left(K_{i} \neq K_{d}\right)$ the isotherms and thus the isopycnals must meet the bottom boundary at right angles.

If one takes the geothermal heat flux $F_{\text {geo }}$ into consideration the boundary condition at the lake bottom for temperature transport is given by: 


$$
\begin{aligned}
& F_{\text {geo }}=\rho \cdot c_{p} \cdot \vec{F}_{r, z}^{T} \cdot \vec{e}_{s}=\rho \cdot c_{p} \cdot K_{d}\left(-\frac{\partial T}{\partial r} \sin \gamma+\frac{\partial T}{\partial z} \cos \gamma\right) \\
& \Rightarrow \frac{\partial T}{\partial r} \sin \gamma=-\frac{F_{\text {geo }}}{\rho \cdot c_{p} \cdot K_{d}}+\frac{\partial T}{\partial z} \cos \gamma \cdot
\end{aligned}
$$

where $c_{p}$ is the specific heat at constant pressure. The geothermal heat flux $F_{\text {geo }}$ is assumed to be spatially and temporally constant. Its dependence on water temperature is neglected.

Note that the boundary conditions for passive tracers and temperature can have a different form. For example, if the bottom boundary is impermeable for the tracer its flux perpendicular to the bottom boundary must vanish. Since the flux of a passive tracer is given by equation 7 the isopycnal diffusion coefficient plays a role in the boundary condition. As a consequence isopycnal mixing influences the vertical distribution of the tracer even far from the boundaries. Therefore, the effective vertical diffusion coefficient $K_{z \text {,eff }}$ determined from the temporal development of the vertical variance $\sigma_{z, \text { tracer }}^{2}$ of a tracer cloud:

$$
K_{z, \text { eff }}=\begin{array}{cc}
1 & \partial \sigma_{z, \text { tracer }}^{2} \\
2 & \partial t
\end{array}
$$

does not necessarily agree with the diapycnal diffusion coefficient.

The upper boundary of the modelled water body can be regarded as the transition between epilimnion and hypolimnion. The epilimnion is supposed to act as a heating plate so that the temperature at the upper boundary is the temperature of the epilimnion. The depth of the epilimnion is assumed to be constant.

Since the lake as well as the initial and boundary conditions are radially symmetric isotherms and isopycnals have to be horizontal in the middle of the lake (at $r=0)$ :

$$
\begin{aligned}
& \partial T \\
& \partial r
\end{aligned}=0 \text { thus } \beta(0, z)=90^{\circ} .
$$

The only requirement for the temperature distribution at time $t=0$ is that it has to be radially symmetric.

\section{d) Numerical implementation}

The model equation (equation 13) was solved numerically with the method of lines. The partial differential equation was transformed into a set of coupled ordinary differential equations by approximating the spatial derivatives using 2 nd order Taylor expansion. The points at the sloping boundary were accounted for by considering additional neighbouring points. The grid size was chosen to provide a resolution of $0.2 \mathrm{~m}$ in the vertical and of $5 \mathrm{~m}$ in the horizontal. At locations were the slope of the lake boundary is large the horizontal resolution was increased to guarantee the vertical resolution of $0.2 \mathrm{~m}$. The set of ordinary differential equations was solved using the algorithm of Hindmarsh (1980). 


\subsection{Morphometry and parameters used in the model}

The shape of the basin was modelled from the morphometry of Lake Alpnach. A radially symmetric basin was constructed by assuming the cross-sections in different depths to be radially symmetric. From the radius corresponding to the area of the particular cross-sections the angle $\gamma(r, z)$ could be calculated.

The geothermal heat flux was estimated to be $F_{\text {geo }}=0.12 \mathrm{~W} \mathrm{~m}^{-2} \pm 10 \%$ by averaging measurements of the geothermal heat flux from Lake Lucerne (Finckh, 1981), to which Lake Alpnach is directly connected. Finckh (1981) determined $F_{\text {geo }}$ from the subbottom temperature gradient down to a maximum depth of $10.5 \mathrm{~m}$ below the lake bottom using a special corer. The distance between measuring sites and Lake Alpnach is about $10 \mathrm{~km}$. To analyse the influence of the geothermal heat flux on the development of a boundary layer we have run the model with $F_{\text {geo }}=0.12 \mathrm{~W} \mathrm{~m}^{-2}$ and without geothermal heating $\left(F_{\text {geo }}=0\right)$.

To show the principal consequences of the model the upper boundary condition (epilimnion) was modelled by a sinusoidal increase in temperature beginning at $5^{\circ} \mathrm{C}$ and reaching $20^{\circ} \mathrm{C}$ after 120 days $(T=5+15 \cdot \sin (t \cdot \pi / 240)$ where $t$ is time in days). Beginning with $t=0$ at April 10th this boundary condition reaches a maximum temperaure of $20{ }^{\circ} \mathrm{C}$ in August. It provides temperatures which agree within $1{ }^{\circ} \mathrm{C}$ with our measurements from $5 \mathrm{~m}$ depth taken on May 13th, July 22nd and October 15 th. Initial temperatures were assumed to be $5{ }^{\circ} \mathrm{C}$ everywhere in the lake, which is a typical situation in spring.

The diapycnal diffusion coefficient was assumed to be constant spatially and temporally. Using the heat budget method (see Powell and Jassby, 1974) apparent vertical diffusion coefficients were calculated from our CTD profiles measured in May, July and October 1992. For the period from mid May to mid October we obtained apparent vertical diffusivities ranging from $1 \cdot 10^{-6}$ and $5 \cdot 10^{-6} \mathrm{~m}^{2} \mathrm{~s}^{-1}$ depending on depth (depth region between 10 and $33 \mathrm{~m}$ ). For the period between $22 \mathrm{nd}$ of July and 15th of October 1992 and in the depth range between 15 and $30 \mathrm{~m}$ the values averaged at about $1.5 \cdot 10^{-6} \mathrm{~m}^{2} \mathrm{~s}^{-1}$. The values of the vertical diffusivities observed by us in 1992 are in good agreement with vertical diffusivities of $2 \cdot 10^{-6}$ to $3 \cdot 10^{-6} \mathrm{~m}^{2} \mathrm{~s}^{-1}$ determined from the vertical spread of an artificial tracer, from the heat budget method and from microstructure measurements in Lake Alpnach in 1989 (Wüest et al., 1996b).

To demonstrate the effect of the diapycnal diffusivity on the development and the structure of the boundary layer we have run the model using diffusivities of $1 \cdot 10^{-6}, 3 \cdot 10^{-6}$, and $5 \cdot 10^{-6} \mathrm{~m}^{2} \mathrm{~s}^{-1}$, thus covering the range of values observed experimentally.

\section{Model results and discussion}

The simple diffusion model predicts the development of a distinct boundary layer (Fig. 5). The results presented in Fig. 5 were calculated with a diapycnal diffusion coefficient of $K_{d}=3 \cdot 10^{-6} \mathrm{~m}^{2} \mathrm{~s}^{-1}$. The small pictures (left in Fig. 5) show the modelled basin up to its maximum depth where the large pictures illustrate the structure of the boundary layer in more detail. For short diffusion times this layer is comparably thick (Fig. 5a) due to the influence of the geothermal heat flux. For 

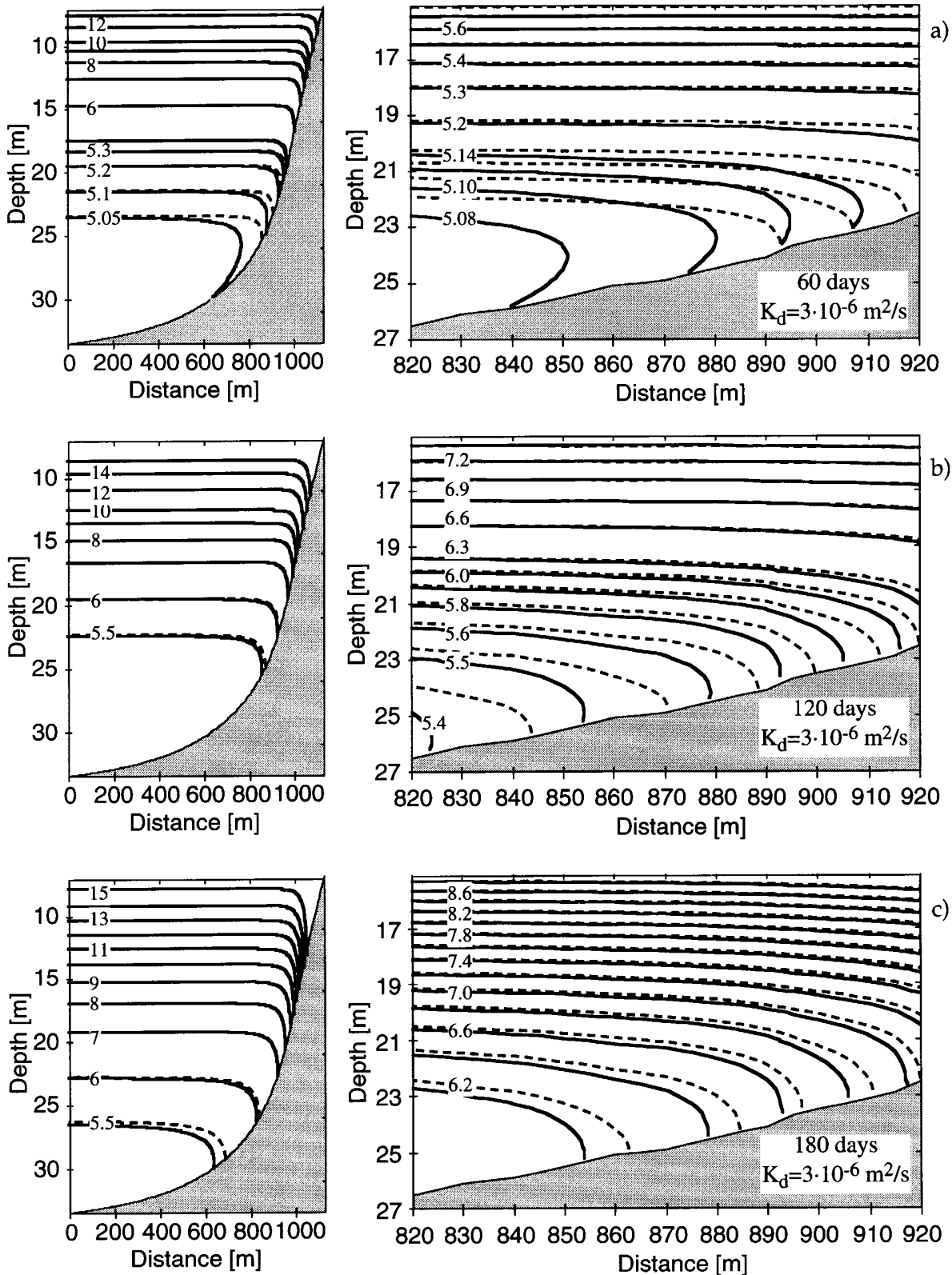

Figure 5. Prediction of the simplified diffusion model for the temporal development of the temperature distribution for a diapycnal diffusion coefficient of $3 \cdot 10^{-6} \mathrm{~m}^{2} \mathrm{~s}^{-1}$ and a geothermal heat flux of $F_{\text {geo }}=0.12 \mathrm{~W} \mathrm{~m}^{-2}$. Small pictures show the modelled basin up to its maximum depth while the large pictures give a more detailed view of the boundary region. The dashed line show the model results for $F_{\text {geo }}=0$ 
a)

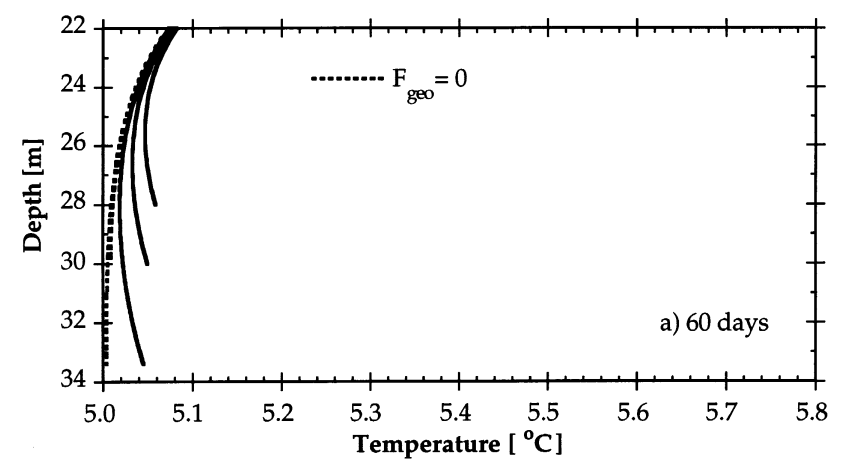

b)

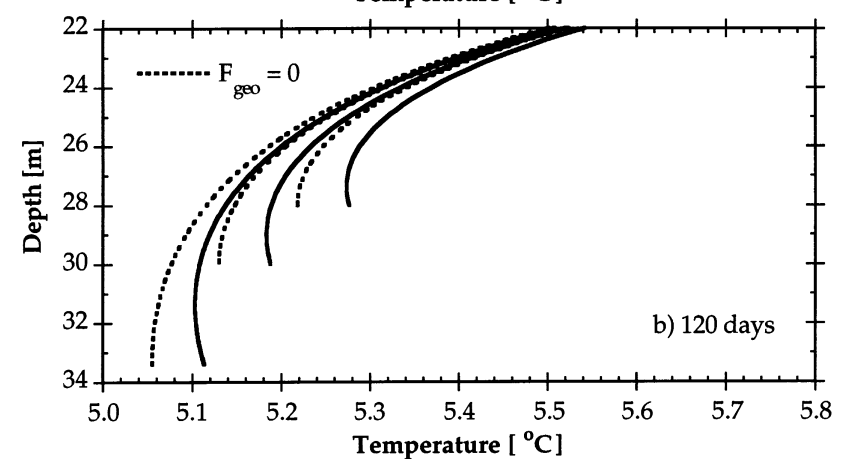

c)

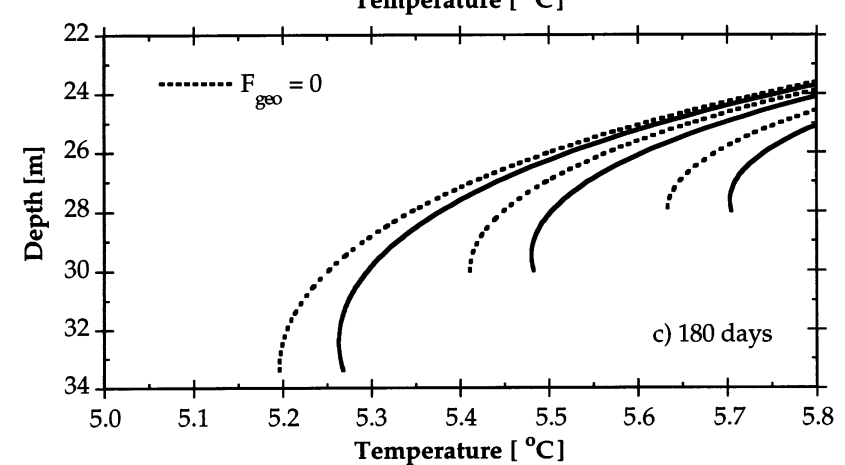

Figure 6. Vertical temperature profiles predicted by the simplified diffusion model for diffusion times of 60 days (a), 120 days (b) and 180 days (c) using a diapycnal diffusion coefficient of $3 \cdot 10^{-6} \mathrm{~m}^{2} \mathrm{~s}^{-1}$ and a geothermal heat flux of $F_{\text {geo }}=0.12 \mathrm{~W} \mathrm{~m}^{-2}$. The dashed lines show the model results for $F_{\text {geo }}=0$. The three profiles in each simulation stem from different locations $(0 \mathrm{~m}, 620 \mathrm{~m}$ and $750 \mathrm{~m}$ distance from the deepest point of the lake)

larger diffusion times the transport of heat from the epilimnion and thus the transport of density towards it becomes dominant and the thickness of the boundary layer decreases. The thickness of this layer is of the order of 1 to $2 \mathrm{~m}$ which agrees with the eperimental findings.

Profiles of the modelled temperature distribution from different locations indicate that the boundary layer increases with depth of the bottom boundary (Fig. 6). 
This is consistent with our measurements. In the deepest parts of the lake the modelled temperature and density distribution, respectively, is inversely stratified especially for short diffusion times. Since advective processes are neglected in the model, convection cannot occur. However, convection would homogenise the temperature and the density distribution in the vertical and cause the transition from the boundary layer to the open water to sharpen. This would improve the agreement between model and experiment. Note that the temperature and density gradients in the inversely stratified region near the bottom boundary are small, especially for long diffusion times.

The role of the geothermal heat flux is shown in Figs. 5 and 6. The results of the model with $F_{\text {geo }}=0$ (dashed lines) are compared to the results with a flux of $F_{\text {geo }}=0.12 \mathrm{~W} \mathrm{~m}^{-2}$. Even if the flux of geothermal heat is zero a boundary layer develops. The thickness of this boundary layer appears to be smaller than that for a heat flux of $0.12 \mathrm{~W} \mathrm{~m}^{-2}$ (Fig. 5).

The inverse stratification discussed above vanishes if the geothermal heat flux is zero (Figs. 5 and 6). Since the initial temperature is assumed to be constant in the entire basin, near the bottom boundary (especially in deep regions of the lake) the heat flux due to geothermal heat is large compared to the heat flux from the epilimnion for short diffusion times or small diffusion coefficients. Thus, the water near the lake boundary is warmer and less dense than the water above it. The stratification is inverse. For longer diffusion times the heat flux from the epilimnion dominates the geothermal heat flux and thus the gradients in temperature and density become positive. The deeper the lake boundary the longer it takes until the heat flux from the epilimnion is large compared to the geothermal heat flux. Therefore geothermal heat especially influences the thickness of the boundary layer in the deeper parts of the lake.

The influence of the magnitude of the diapycnal diffusion coefficient on the density distribution is demonstrated in Fig. 7. The diffusion time is 120 days. The geothermal heat flux is $F_{\text {geo }}=0$. In all cases shown $\left(K_{z}=1 \cdot 10^{-6} \mathrm{~m}^{2} \mathrm{~s}^{-1}, K_{z}=3 \cdot 10^{-6} \mathrm{~m}^{2} \mathrm{~s}^{-1}\right.$ and $K_{z}=5 \cdot 10^{-6} \mathrm{~m}^{2} \mathrm{~s}^{-1}$ ) a boundary layer develops. The thickness of this layer increases with increasing diffusion coefficient. For $K_{z}=5 \cdot 10^{-6} \mathrm{~m}^{2} \mathrm{~s}^{-1}$ the layer is about 2 times larger than for $K_{z}=1 \cdot 10^{-6} \mathrm{~m}^{2} \mathrm{~s}^{-1}$.

In the following we describe a model run with a modified set of initial and boundary conditions. The initial temperature distribution for the model was taken from the CTD measurements of the 13th of May. Since the experimental data were not sufficient to reconstruct the three-dimensional temperature distribution the vertical temperature profile from the centre of the lake was taken as representative for all locations. This assumes horizontal homogeneity in May. As upper boundary condition served the temperature at $18 \mathrm{~m}$ depth measured in May, July, October and November. The temporal development of the temperatures in $18 \mathrm{~m}$ depth was obtained by linear interpolation between the measurements. The depth chosen for the upper boundary condition corresponds to the epilimnion depth in November at the site of our measurements. The model includes a geothermal heat flux of $F_{\text {geo }}=0.12 \mathrm{~W} \mathrm{~m}^{-2}$. Using a diffusion coefficient of $1 \cdot 10^{-6} \mathrm{~m}^{2} \mathrm{~s}^{-1}$ the model was run beginning at the 13th of May to predict the temperature distribution at the 6th of November. The agreement between model predictions and experimental data is surprisingly good (Figs. 4 and 8 ). 

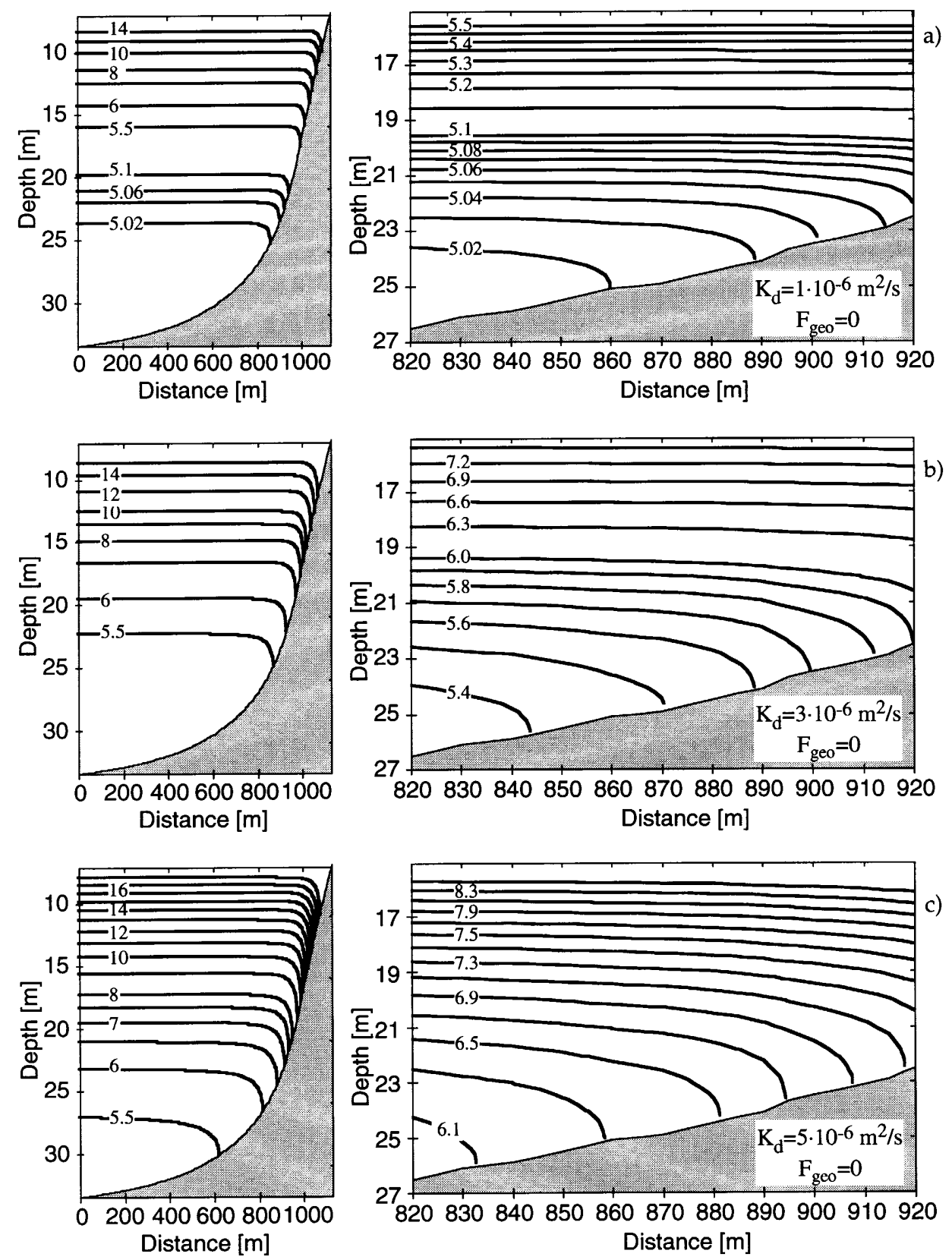

Figure 7. Influence of the diapycnal diffusion coefficient on the density distribution predicted by the model for diffusion times of 120 days. The influence of geothermal heat flux is neglected $\left(F_{\text {geo }}=0\right)$ 

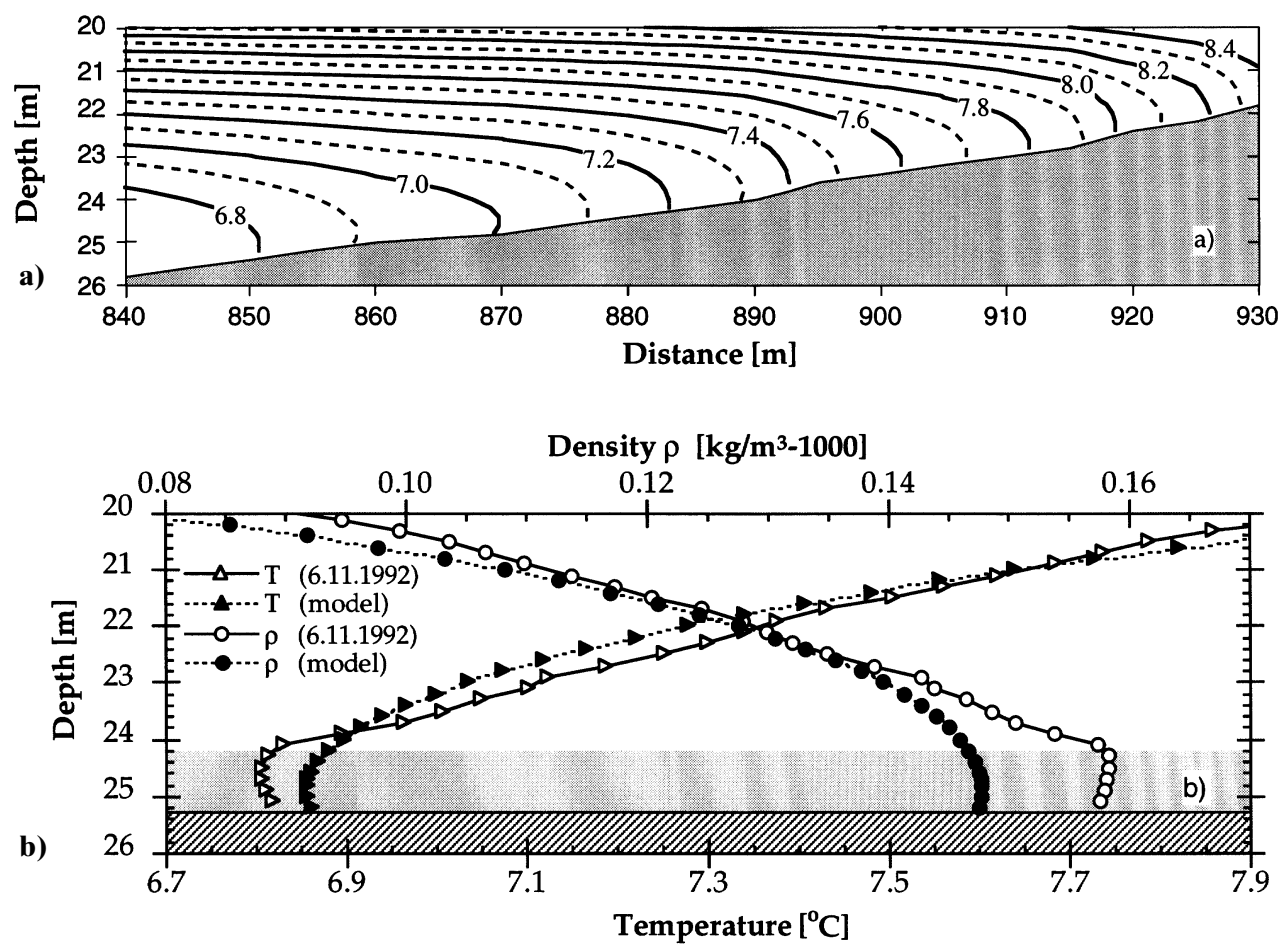

Figure 8. a) Prediction of the diffusion model for the two dimensional temperature distribution in Lake Alpnach. For the initial condition we used a temperature profile taken on 13th of May. Temperatures measured in $18 \mathrm{~m}$ depth throughout the year served as upper boundary condition. b) A comparison between predicted and measured temperature and density profiles on the 6th of November

\section{Limitations of the model}

The most stringent limitations of the model result from the fact that advective processes are not considered. In the model the inversely stratified density profiles, which occur at short diffusion times and for small diffusivities, cannot result in convection. This convection would homogenise the density and temperature distribution in the vertical. This should cause a sharpening of the transition between the boundary layer and the region above it, similar to the sharpening of the transition between epilimnion and hypolimnion during convective deepening of the epilimnion in fall.

Horizontal density gradients near the lake boundary give rise to a secondary circulation as pointed out first by Phillips (1970) and Wunsch (1970). Secondary circulation and the resulting complex current field should alter the structure of the boundary layer. For example, the transition from boundary layer to interior is sharper in the data than in the model output. This could not only be caused by convection as discussed above but also by the re-stratifying influence of secondary circulation. Since our model is purely diffusive, secondary circulation, the complex current field resulting from secondary circulation and its interaction with the mixing 
process which is still discussed controversial (Garrett, 1990) are beyond the scope of our model.

Gloor et al. (1994) have shown that the thickness of the boundary layer in Lake Alpnach can change within a couple of hours due to the motion of internal seiches. From the vertical distribution of temperature and suspended particles they inferred that vertical mixing within the boundary layer is large. Gloor et al. (1994) suggest that the short term change in boundary layer thickness is not caused by an increased mixing near the bottom boundary of the lake but is the result of advection induced by the internal seiche. Neither the seiche motion nor an increase in mixing near the boundary are considered in our model. Note however, that the homogeneous vertical distribution of suspended particles within the boundary layer does not imply an increase in diapycnal mixing but can be explained by our model as will be shown in the next section.

The isopycnal and diapycnal diffusion coefficients were assumed to be constant in time. Temporal varying diffusion coefficients could easily be incorporated into the model. Temporal variation of the diffusion coefficients might improve the agreement between model and experimental results. However, the simple model employed in this work using constant diffusion coefficients is sufficient to predict a boundary layer the structure of which is similar to the observed one.

\section{Implications of the model}

Isopycnal diffusion coefficients are considerably larger than diapycnal diffusion coefficients. In the hypolimnion of lakes $K_{i}$ typically is of the order $1 \cdot 10^{-2} \mathrm{~m}^{2} \mathrm{~s}^{-1}$ (Peeters et al., 1996) whereas $K_{d}$ is about $1 \cdot 10^{-6}$ to $5 \cdot 10^{-6} \mathrm{~m}^{2} \mathrm{~s}^{-1}$. Consequently the transport parallel to the isopycnal must be much larger than perpendicular to it.

The measurements have shown that within the boundary layer the isopycnals are almost parallel to the vertical direction. According to our model the vertical transport of substances in the boundary layer is then determined by the isopycnal diffusion coefficient. Consequently the vertical diffusive flux of chemical substances or particles should be large, even if the gradient of the concentration is small. This result of the model can be employed to interpret the homogeneity of the measured concentration of dissolved particles in the boundary layer. In general, the ratio of the settling velocity and the vertical component of the diffusive flux determines the particle distribution. Assuming steady state conditions and the diffusion tensor to be diagonal in the Cartesian coordinate system near the lake boundary the distribution of suspended particles is given by:

$$
{ }_{\nu}^{\partial d} \partial C_{p}=K \begin{aligned}
& \partial^{2} C_{p} \\
& \partial d^{2}
\end{aligned} \Rightarrow C_{p}(d)=C_{p}(0) e^{-d \cdot v / K},
$$

where $d$ is the distance from the sediment, $K$ the vertical diffusion coefficient and $v$ the settling velocity. According to Stoke's law the latter is smaller than $1 \mathrm{~mm} \mathrm{~s}^{-1}$ for quartz particles with a diameter of less than $40 \mu \mathrm{m}$. As discussed above vertical diffusion within the boundary layer can be approximated by the isopycnal diffusion coefficient. Thus, the exponent in equation 19 is small $\left(v / K<0.1 \mathrm{~m}^{-1}\right)$ and $C_{p}(d)$ decreases only by $10 \%$ per meter distance to the sediment. As result the vertical particle distribution should be approximately constant within the boundary layer. 
Outside this layer the diapycnal diffusion coefficient applies. In this case the exponent is large $\left(v / K>1000 \mathrm{~m}^{-1}\right)$ and $C_{p}(d)$ is small. This is compatible with the measurement of turbidity the logarithm of which is approximately proportional to the concentration of suspended particles. Within the boundary layer turbidity was found to be almost constant and large compared to the region above it (Fig. 2).

According to our model the transport parallel to the lake boundary is caused by diapycnal mixing and therefore should be comparatively small. If, as in our model, pure diffusion is considered the concentration of a substance punctually introduced near the lake sediments should only very slowly spread upslope parallel to the lake boundary within the boundary layer. However, secondary circulation not considered in our model is characterised by an upslope current parallel to the lake boundary and thus particularly influences the transport in this direction. Consequently, tracer experiments could be employed to study the role of secondary circulation in relation to pure diffusion.

\section{Conclusions}

The decrease in temperature and density gradients as well as the vertically almost homogeneous distribution of suspended particles and dissolved substances near a lake boundary can be described without requiring an increase in the diapycnal diffusion coefficient in the boundary layer. A simple diffusion model with constant isopycnal and diapycnal diffusion coefficients provides a good description of the development of a boundary layer, the structure of which (e.g. the boundary layer thickness and the increase of this thickness with depth) is similar to that experimentally observed.

Provided that the isopycnal and diapycnal diffusion coefficients are spatially constant, the model implies that isospycnal mixing has no influence on the density distribution. This means that the structure of the density distribution, in particular the existence of the boundary layer, is only determined by diapycnal mixing and the initial and boundary conditions.

The model suggests that within the boundary layer, the vertical transport of passive tracers is determined by isopycnal diffusion, whereas diapycnal diffusion is responsible for transport parallel to the bottom boundary of the lake. Then, mixing is not isotropic within the boundary layer but large in the vertical direction and small parallel to the lake boundary.

Since advection is not considered in our model it does also not include secondary circulation which causes an upslope transport parallel and close to the lake boundary. Thus, the observation of transport and spread of tracers upslope, parallel and close to the bottom boundary might provide an experimental basis in studies concerned with the role of secondary circulation in relation to pure diffusion for the development of a boundary layer in lakes.

\section{ACKNOWLEDGEMENTS}

The authors would like to express their thanks to M. Schurter for the excellent support in the field. We also thank D. M. Imboden and A. Wüest for their comments on the manuscript. Financial support for this research was made available by the Swiss National Science Foundation grants no. 20-32700.91 and NF-20-36364.92. 


\section{REFERENCES}

Armi, L., 1978. Some evidence for boundary mixing in the deep ocean. J. Geophys. Res. 83:1971-1979.

Carter, H. H. and A. Okubo, 1965. A study of the physical processes of the movement and dispersion in the Cape Kennedy area. Final Rep. under the U.S. Atomic Energy Comm., Cheasapeake Bay Inst., Johns Hopkins Univ., Rep. No. NYO-2973-1, pp. 164.

Chen, C. T. and F. J. Millero, 1986. Precise thermodynamic properties for natural waters covering only the limnological range. Limnol. Oceanogr. 31:657-662.

Ericksen, C. C., 1985. Implications of ocean bottom reflections for internal wave spectra and mixing. J. Phys. Oceanogr. 15:1145-1156.

Finckh, P., 1981. Heat-flow measurements in 17 perialpine lakes: Summary. Geological Society of America. Bulletin, part I, 92:108-111.

Garrett, C., 1990. The role of secondary circulation in boundary mixing. J. Geophys. Res. 95:3181-3188.

Garrett, C., P. MacCready and P. Rhines, 1993. Boundary mixing and arrested Ekman layers: rotating stratified flow near a sloping boundary. Ann. Rev. Fluid Mech. 25:291-323.

Gloor, M., A. Wüest and M. Münnich, 1994. Benthic boundary mixing and resuspension induced by internal seiches. Hydrobiologic 284:59-68.

Hindmarsh, A. C., 1980. LSODE and LSODI, two new initial value ordinary differential equation solvers. ACM-Signum Newsletter 15:10-11.

Ivey, G. N., 1987. The role of boundary mixing in the deep ocean. J. Geophys. Res. 92: $11873-11878$.

Münnich, M., A. Wüest and D. M. Imboden, 1992. Observation of the second vertical mode of the internal seiche in an alpine lake. Limnol. Oceanogr. 37:1705-1719.

Pedlosky, J., 1987. Geophysical fluid dynamics. Springer Verlag, New York.

Peeters, F., A. Wüest, G. Piepke and D. M. Imboden, 1996. Horizontal mixing in lakes. J. Geophys. Res. 101:18361-18375.

Phillips, O. M., 1970. On flows induced by diffusion in a stably stratified fluid. Deap-Sea Res. $17: 435-443$.

Powell, T. and A. Jassby, 1974. The estimation of vertical eddy diffusivities below the thermocline in lakes. Water Resour. Res. 10:191-198.

Redi, M. H., 1982. Oceanic isopycnal mixing by coordinate rotation. J. Phys. Oceanogr. $12: 1154-1158$.

Woods, A. W., 1991. Boundary-driven mixing. J. Fluid. Mech. 226:625-654.

Wunsch, C., 1970. On oceanic boundary mixing. Deep-Sea Research 17:293-301.

Wüest, A., G. Piepke and J. D. Halfman, 1996a. Combined effects of dissolved solids and temperature on the density stratification of Lake Malawi. In: T. D. Johnson and E. O. Odada (eds.): The Limnology, Climatology and Paleoclimatology of the East African Lakes. Gordon and Breach.

Wüest, A., D. C. Van Senden, J. Imberger, G. Piepke and M. Gloor, 1996b. Comparison of diapycnal diffusivity measured by tracer and microstructure techniques. Dyn. Atmosph. Oceans $24: 27-39$.

Received 28 November 1995;

revised manuscript accepted 19 December 1996. 